\title{
A hospital-based study of prophylactic therapy in glucocorticoid-induced osteoporosis
}

\section{S Lekamwasam ${ }^{1}$}

(Index words: osteoporosis, bone mineral density, glucocorticoids)

\begin{abstract}
Objective To find out the proportion of patients who qualifies to receive prophylactic therapy for glucocorticoidinduced osteoporosis.
\end{abstract}

Design Retrospective record review.

Participants Current users of oral glucocorticoids referred for bone mineral density estimation to assess their fracture risk $(n=134)$.

Measurements Clinical history and bone mineral density of the spine and proximal femur.

Results Based on the current UK guidelines published by the College of Physicians of London in 2002, 22 of 57 (probability of 0.39 ) patients under 20 years, 19 of 38 (probability of 0.5 ) between 20-49 years, 22 of 28 (probability of 0.79 ) between 50-64 years and 10 of 11 (probability of 0.9 ) above 64 years, qualified for the diagnosis of glucocorticoid-induced osteoporosis and prophylactic therapy was indicated for them. The prevalence of glucocorticoid-induced osteoporosis was not different between men and women in any age group.

Conclusions Due to the restricted availability of DXA scan facility, initiation of prophylactic therapy without baseline

${ }^{1}$ Centre for Metabolic Bone Diseases, Faculty of Medicine, Galle, Sri Lanka.

Correspondence: SL, e-mail <sarathlk@sltnet.lk>. Received 14 July 2009 and revised version accepted 5 February 2010. Competing interests: none declared. 
bone mineral density appears rational in current users of oral glucocorticoids older than 50 years as $80-90 \%$ of them would qualify for such therapy. However, only $40-50 \%$ of current glucocorticoids users younger than 50 years would require such therapy and simultaneous prescribing of prophylaxis appears unnecessary in $50-60 \%$ of them. Attempts should be made to estimate baseline bone mineral density in this group of patients.

\section{Introduction}

Glucocorticoids (GCs) are used in many inflammatory and non-inflammatory diseases, often, for prolonged periods. Prolonged use of systemic GCs is associated with bone loss, predisposing them to fractures [1]. Approximately $30 \%$ of bone loss associated with GC use occurs within the first 3-6 months after initiation of therapy [2]. The bone loss associated with GC use is dose dependent and related to underlying co-morbidity [3]. Furthermore, patients on GCs fracture at a higher bone mineral density (BMD) than postmenopausal women [4]. Hence, a T score higher than that used to define postmenopausal osteoporosis is used to diagnose glucocorticoid-induced osteoporosis (GIO) [5]. Current management guidelines categorise the fracture risk of patients on GCs, based on their age and other clinical manifestations. The current UK guidelines published by the College of Physicians of London use age and previous fracture history to determine the fracture risk of patients on GCs [5].

According to the UK guidelines, simultaneous use of prophylaxis to prevent GC-induced bone loss is recommended for high-risk patients; patients older than 65 years and those who have already suffered fractures. Patients younger than 65 years with no fracture, form a low-risk category and they need to have BMD measured and prophylaxis is recommended only if their BMD, either in the spine or hip, is found to be 1.5 standard deviations below the mean of the normal reference population [5].

In Sri Lanka, the availability of central-type DXA is limited and clinicians have a dilemma in treating patients, especially those in the low-risk category. It is often questioned whether simultaneous prophylaxis can be initiated in all patients who are considered for long-term GC therapy, irrespective of their age, BMD and other risk factors. This type of therapeutic approach cannot be justified as the bone loss associated with long term GC use has a considerable heterogeneity, probably mediated through genetic factors such as vitamin D and GC-receptor polymorphism [2]

In this study, we analysed all current users of GCs referred for BMD evaluation to the Centre for Metabolic Bone Diseases, in Galle. These patients were referred by clinicians to assess the future fracture risk as a part of their routine medical management.

\section{Methods}

Medical records of all patients who have been referred for BMD evaluation to our DXA facility (Hologic Discovery by Hologic Inc, MA, USA) in Galle were extracted. These patients have been on prednisolone more than $5 \mathrm{mg}$ per day for a minimum period of three months prior to DXA evaluation and none had received specific treatment given for osteoporosis. Patients with history of asthma were excluded from the analysis. Ten patients were on pharmacological doses of calcium with or without vitamin $\mathrm{D}_{3}$ (maximum of $200 \mathrm{IU}$ per day). All patients had undergone BMD estimation of total lumbar spine (L1 to L4 in postero-anterior projection) and proximal right femur. One technician performed all scans adhering to the guidelines given by the DXA manufacturer. Some children required mild sedation to position them correctly.

The local guidelines allow free DXA scanning for any patient who is on systemic GCs or who is considered for long term GCs. All clinicians have a direct access to this facility and most of the scans are done on the same day or on a day convenient to the patient. Scanner is located in the ground floor of the main entrance hall and easily accessible. Patients are referred routinely and patients who were not referred for DXA are unlikely to be systematically different from those who were referred.

Furthermore, only those who had taken prednisolone minimum of $5 \mathrm{mg}$ per day for a minimum of 3 months, consecutively, were selected for this study. This type of conservative approach was adopted as current guidelines and most of clinical trials are applicable for those who are currently on GCs [5].

Age of the patients ranged from 10 to 73 years and none had suffered either vertebral or non-vertebral fracture previously. Patients were categorised according to their age; $10-19(\mathrm{n}=57), 20-49(\mathrm{n}=38), 50-64(\mathrm{n}=28)$ and 65 years and over $(\mathrm{n}=11)$. In patients older than 20 years, $\mathrm{T}$ score of total spine and total hip were calculated using young adult Asian reference data provided by the manufacturer and those who had $\mathrm{T}$ score equal or less than -1.5 in either site, were considered having glucocorticoid-induced osteoporosis [5]. In patients below 20 years, Z score was calculated for the same two skeletal sites using age and sex-matched reference data provided by the manufacturer and those who had $\mathrm{Z}$ score equal or less than -2.0 were considered having GIO [6].

\section{Results}

Of 57 patients under 20 years, 22 had evidence of GIO (probability and odds ratio of GIO are 0.39 and 0.63 , respectively). Out of 38 patients between $20-49$ years, 19 (probability and odds ratio of GIO are 0.50 and 1.0, respectively) qualified for the diagnosis of GIO. Furthermore, 22 out of 28 patients (probability and odds ratio of GIO are 0.79 and 3.7, respectively) between 50-64 years and 10 of 11 patients (probability and odds ratio of 
GIO are 0.90 and 10, respectively) above 65 years showed evidence of GIO. There was no statistically significant difference in the prevalence of GIO between men and women in any age group.

\section{Discussion}

Our data, in a relatively small sample of patients who have taken daily dose of prednisolone more than $5 \mathrm{mg}$ for a minimum of three months, re-emphasise the management guidelines given by the UK guideline group. According to the guidelines, patients over 65 years are considered a high-risk category, and simultaneous use of prophylaxis is recommended for them [5]. In our analysis, 10 out of 11 (probability of 0.90) in this age group had GIO and this justifies the use of simultaneous prophylactic therapy, regardless of their baseline BMD, in them. In patients below 50 years, only $39-50 \%$ of those taking GCs had low BMD to qualify for prophylactic therapy and this again emphasises the need of BMD estimation in this age group to select the correct patient. Owing to the restricted availability of DXA, if all these patients were commenced on simultaneous prophylactic therapy many of them would have received the treatment unnecessarily. Our data, however, indicate that in patients between 50-64 years, the probability of GIO is nearly 0.8 and simultaneous prophylactic therapy in them, regardless of their baseline BMD, appears logical.

Based on these data, we would like to make following recommendations for clinicians who use long term GCs to treat their patients. When patients are older than 65 years, use of prophylactic therapy without BMD estimation is justifiable. When patients are younger than 65 years, attempts should be made to measure hip and spine BMD and prophylactic therapy should only be prescribed to those with low BMD.

If DXA is not available, clinician may consider using prophylactic therapy in all patients above 50 years as they have a high prevalence of the disease. For those who are younger than 50 years, the decision is left to the clinician. If prophylaxis is offered to all patients below 50 years, nearly half of patients between $20-50$ years and $60 \%$ of patients between 10-20 years will receive treatment for no valid reason. In these patients, other clinical measures such as nature of the underlying disease (inflammatory or noninflammatory) and body mass index (BMI) can be used to strengthen the claim for prophylactic therapy. Inflammatory diseases such as rheumatoid arthritis and low BMI are independent determinants of fractures [7].

Although many drugs have been tested in GIO, only alendronate, residronate and teriparatide are found to have anti-fracture efficacy. Of these, only alendronate and residronate are currently licensed for GIO [8]. Although these drugs are generally safe, newer side effects such as osteonecrosis of the jaw and increased incidence of atrial fibrillation [9] have been recognised and the use of bisphosphonates should be justified by the clinical situation.

The cost of therapy versus DXA examination is another aspect the clinician could consider when deciding treatment. One DXA examination in the private sector would cost between 3000-4000 Sri Lankan rupees and this is equal to the cost of one year treatment with weekly generic alendronate.

This analysis has many limitations. Study sample is relatively small and this did not allow us to do a subgroup analysis based on gender and disease category. Furthermore, we were unable to gather the cumulative dose of GCs in these subjects as there was no proper maintenance of records. The cumulative dose of GC is a better reflection of GC induced bone loss [10] and patients could have been categorised based on this information. However, this was a limitation in our study.

\section{References}

1. Kanis JA, Johansson H, Oden A, et al. A meta-analysis of prior corticosteroid use and fracture risk. Journal of Bone and Mineral Research 2004; 19: 893-9.

2. Alesci S, De Martino MU, Ilias I, Gold PW, Chrousos GP. Glucocorticoid-induced osteoporosis: From basic mechanisms to clinical aspects. Neuroimmunomodulation 2005; 12: 1-19.

3. Hela S, Nihel M, Faten L, et al. Osteoporosis and Crohn's disease. Joint, Bone and Spine 2005; 72: 403-7.

4. Van Staa TP, Laan RF, Barton IP, Cohen S, Reid DM, Cooper, C. Bone density threshold and other predictors of vertebral fracture in patients receiving oral glucocorticoid therapy. Arthritis and Rheumatology 2003; 48: 3224-9.

5. Compston J. US and UK guidelines for glucocorticoidinduced osteoporosis: Similarities and differences. Current Rheumatology Reports 2004; 6: 66-9.

6. Anonymous. Position statement: Introduction, methods, and participants. The Writing Group for the International Society for Clinical Densitometry (ISCD) Position Development Conference. Journal of Clinical Densitometry 2004; 7: 13-6.

7. Van Staa TP, Geusens P, Pols HA, de Laet C, Leufkens HG, Cooper C. A simple score for estimating the long term risk of fracture in patients using oral glucocorticoids. Quarterly Journal of Medicine 2005; 98: 191-8.

8. Compston J. Glucocorticoid-induced osteoporosis. Hormone Research 2003; 60 Suppl 3: 77-9.

9. Strampel W, Emkey R, Civitelli R. Safety considerations with bisphosphonates for the treatment of osteoporosis. Drug Safety 2007; 30: 755-63.

10. De Vries F, Bracke M, Leufkens HG, et al. Fracture risk with intermittent high-dose oral glucocorticoid therapy. Arthritis and Rheumatology 2007; 56: 208-14. 